\title{
Antibody with Infinite Affinity for In Vivo Tracking of Genetically Engineered Lymphocytes
}

\author{
Simone Krebs ${ }^{1}$, Afruja Ahad ${ }^{1}$, Lukas M. Carter ${ }^{2}$, Justin Eyquem ${ }^{3}$, Christian Brand ${ }^{2}$, Meghan Bell ${ }^{1}$, Vladimir Ponomarev ${ }^{2}$, \\ Thomas Reiner ${ }^{2}$, Claude F. Meares ${ }^{4}$, Stephen Gottschalk ${ }^{5}$, Michel Sadelain ${ }^{3}$, Steven M. Larson ${ }^{1}$, and Wolfgang A. Weber ${ }^{1,6}$ \\ ${ }^{I}$ Molecular Imaging and Therapy Service, Department of Radiology, Memorial Sloan Kettering Cancer Center, New York, New York; \\ ${ }^{2}$ Radiochemistry and Molecular Imaging Sciences Service, Department of Radiology, Memorial Sloan Kettering Cancer Center, New \\ York, New York; ${ }^{3}$ Center for Cell Engineering and Immunology Program, Sloan Kettering Institute, New York, New York; ${ }^{4}$ Chemistry \\ Department, University of California, Davis, California; ${ }^{5}$ Department of Bone Marrow Transplant and Cellular Therapy, St. Jude Children's \\ Research Hospital, Memphis, Tennessee; and ${ }^{6}$ Department of Nuclear Medicine, Technical University of Munich, Munich, Germany
}

See an invited perspective on this article on page 1892.

There remains an urgent need for the noninvasive tracking of transfused chimeric antigen receptor (CAR) T cells to determine their biodistribution, viability, expansion, and antitumor functionality. DOTA antibody reporter 1 (DAbR1) comprises a single-chain fragment of the antilanthanoid-DOTA antibody 2D12.5/G54C fused to the human CD4-transmembrane domain and binds irreversibly to lanthanoid (S)-2-(4-acrylamidobenzyl)-DOTA (AABD). The aim of this study was to investigate whether DAbR1 can be expressed on lymphocytes and used as a reporter gene as well as a suicide gene for therapy of immune-related adverse effects. Methods: DAbR1 was subcloned together with green fluorescent protein into an SFG-retroviral vector and used to transduce CD3/CD28-activated primary human T cells and second-generation $1928 z$ (CAR) T cells. Cell surface expression of DAbR1 was confirmed by cell uptake studies with radiolabeled AABD. In addition, the feasibility of imaging of DAbR1-positive $T$ cells in vivo after intravenous injection of ${ }^{86} \mathrm{Y} /{ }^{177} \mathrm{Lu}-\mathrm{AABD}$ was studied and radiation doses determined. Results: A panel of DAbR1-expressing T cells and CAR T cells exhibited greater than 8-fold increased uptake of ${ }^{86} Y$-AABD in vitro when compared with nontransduced cells. Imaging studies showed ${ }^{86} Y-A A B D$ was retained by DAbR1-positive $T$ cells while it continuously cleared from normal tissues, allowing for in vivo tracking of intravenously administered CAR T cells. Normal-organ dose estimates were favorable for repeated PET/CT studies. Selective T cell ablation in vivo with ${ }^{177}$ Lu-AABD seems feasible for clustered T-cell populations. Conclusion: We have demonstrated for the first time that $T$ cells can be modified with DAbR1, enabling their in vivo tracking via PET and SPECT. The favorable biodistribution and high image contrast observed warrant further studies of this new reporter gene.

Key Words: DOTA antibody reporter gene; PET imaging; lymphocytes; CAR T cells

J Nucl Med 2018; 59:1894-1900

DOI: 10.2967/jnumed.118.208041

Received Jan. 12, 2018; revision accepted Jun. 7, 2018.

For correspondence or reprints contact: Simone Krebs, Molecular Imaging and Therapy Service, Department of Radiology, Memorial Sloan Kettering Cancer Center, 1275 York Ave., New York, NY 10065.

E-mail: krebss@mskcc.org

Published online Jun. 14, 2018.

COPYRIGHT (c) 2018 by the Society of Nuclear Medicine and Molecular Imaging.
C specific recognition, ativation, and proliferation cognition, activation, and proliferation independent of major histocompatibility complex (1-3). Successful treatment of B-cell malignancies with CD19-CAR T cells was a milestone for this approach $(4,5)$. However, the success of CAR T-cell therapy for solid tumors has been limited so far, and the mechanisms for the tumor's treatment resistance are not well understood. Current methods used to predict or monitor the activity of infused $\mathrm{T}$ cells in patients provide limited data on treatment efficacy $(6,7)$. The ability to map the physical distribution and expansion of adoptively transferred $\mathrm{T}$ cells throughout the body in a longitudinal manner could therefore significantly improve real-time monitoring of T-cell activity against tumors, reveal potential toxicity from off-tumor-site targeting, and contribute to exploring adjuvant therapies to enhance adoptive T-cell efficacy. Moreover, in vivo monitoring of engineered T-cell proliferation and persistence will provide crucial information for better and safer clinical protocols and accelerated regulatory approval.

Reporter gene imaging with PET or SPECT appears best suited to noninvasively and quantitatively study the location, viability, and function of transplanted cells in vivo. Stable expression of a reporter gene enables serial longitudinal imaging of cell trafficking, and as the reporter gene is passed on to the progeny cells, imaging of expanding cell populations is possible, thereby providing information about cell viability $(8,9)$. PET and SPECT coregistered with CT enable noninvasive, repetitive detection of radiotracer molecules at picomolar concentrations and high spatial anatomic resolution. Thus, these imaging modalities can provide exquisite sensitivity and specificity for detection of CAR T cells, once a CAR T-cell-specific reporter gene with high binding affinity for a molecular imaging agent and no expression in normal tissues has been developed.

Currently, herpes simplex virus-1 thymidine kinase (HSV-tk) and its mutant HSV-sr39tk are used to track cells in clinical investigational protocols (10). Both HSV-tk and HSV-sr39tk are viral proteins; immune reactions remain a serious concern. Indeed, immune responses have been reported in patients who received anti-HIV cytotoxic HSV-tk transduced T cells (11). To avoid immunogenic responses, naturally expressed reporter genes have been evaluated, including human sodium iodide symporter (12), human norepinephrine transporter (hNET) (13), and human deoxycytidine kinase-based reporter genes (14). These reporter genes may avoid the problem of immunogenicity; however, their physiologic expression hinders successful clinical translation. 
To overcome the current limitations of tracking $\mathrm{T}$ cells in vivo, we explored the feasibility of using cell surface-bound antibody fragments as reporter genes that can bind covalently to small DOTA haptens, thereby acting as reporter probes for PET or SPECT imaging. The specific antibody fragment we tested is based on the antibody 2D12.5 developed by Goodwin and Meares, which binds DOTA-chelates of lanthanoids $(15,16)$. Corneillie et al. (17) introduced cysteine at position 54 of this antibody (2D12.5/G54C) to allow for a covalent bond between the acrylamide group of (S)-2-(4-acrylamidobenzyl)DOTA (AABD) and the antibody $(18,19)$. We have previously conjugated a single-chain fragment of 2D12.5/G54C to CD4 for anchoring it at the cell surface and named the resulting gene DOTA antibody reporter 1 (DAbR1) (20). The aim of the present study was to investigate the feasibility of using DAbR1 as a reporter gene for in vivo tracking of genetically engineered lymphocytes.

\section{MATERIALS AND METHODS}

\section{Design and Assembly of DAbR1 Gene}

DAbR1, which consists of the single-chain Fv fragment of the anti-YDOTA antibody 2D12.5/G54C including human IgG4-CH2CH3 spacer and human T-cell CD4-transmembrane domain, was subcloned together with a P2A element and the gene for green fluorescent protein (GFP) into an SFG-retroviral vector. In addition, an SFG-retroviral vector encoding for 1928z (CD19 CAR) was used (Fig. 1A). Cloning was verified by sequencing (Integrated Genomics Operation, MSK). Transduction was confirmed by flow cytometric analysis using GFP (or $\left.\mathrm{F}(\mathrm{ab})_{2}\right)$. Nontransduced, DAbR1, and CAR-DAbR1 T cells included CD4- and CD8-positive subpopulations and effector memory (CD62 L-negative CD45 RA-negative) as well as effector (CD62 L-negative CD45 RA-negative) T cells.

\section{Retrovirus Production and Generation of DAbR1, $1928 z$} (CAR), and 1928z-DAbR1 (CAR-DAbR1) T Cells

Plasmids encoding the SFG $\gamma$-retroviral vector (21) were prepared as previously described (22). Vesicular stomatitis virus G-protein pseudotyped retroviral supernatants derived from transduced gpg29 fibroblasts (H29) were used to construct stable retrovirus-producing cell lines as previously described $(21,22)$. A general protocol to transduce $\mathrm{T}$ cells with retroviral vectors has been described in detail (23). To generate CAR-DAbR1 T cells, simultaneous transduction with both vectors was performed.

\section{Blood Donors and Cell Lines}

Buffy coats from healthy volunteer donors were obtained from the New York Blood Center. The U373 glioma cell line was a gift from Stephen Gottschalk and the Nalm-6 cell line was a gift from Michel Sadelain, maintained at $37^{\circ} \mathrm{C}$ in RPMI (Thermo Fisher Scientific HyClone; Lonza) containing 10\% (v/v) fetal bovine serum. Cell lines were transduced to express firefly luciferase-GFP $(21,23)$.

\section{Flow Cytometry}

We used an LSR II instrument, FACSdiva software (both from Becton, Dickinson and Co.), and Flowjo software. T cells were analyzed with fluorescein-conjugated monoclonal antibodies directed against antihuman $\mathrm{F}(\mathrm{ab})_{2}$ (Jackson ImmunoResearch) and CD4, CD8, CD62 L, and CD45RA (Becton Dickinson) surface proteins. CAR was labeled with a goat antimouse Fab (Jackson ImmunoResearch, 115-606-003).

\section{Magnetic Activated Cell Sorting}

DAbR1-expressing $\mathrm{T}$ cells were incubated with biotin-conjugated anti- $\mathrm{F}(\mathrm{ab})_{2}$ antibodies (Jackson ImmunoResearch) conjugated with paramagnetic beads and purified following standard magnetic activated cellsorting procedures as specified by the device manufacturer (Miltenyi Biotec).

\section{Cytotoxicity Assays}

The cytotoxicity of $\mathrm{T}$ cells transduced with CD19-CAR was determined by standard luciferase-based assay using NALM-6-expressing firefly luciferase-GFP as target cells (24).

\section{Synthesis and Radiolabeling of AABD}

AABD was prepared following the method of Corneillie et al. (18) and radiolabeled with ${ }^{177} \mathrm{Lu}$ or ${ }^{86} \mathrm{Y}$. ${ }^{177} \mathrm{Lu}$ was purchased from iThemba IDB-Holland as no-carrier-added ${ }^{177} \mathrm{Lu}_{-} \mathrm{LuCl}_{3}$ in $0.1 \mathrm{M}$ $\mathrm{HCl} .{ }^{86} \mathrm{Y}$ (no carrier added, ${ }^{86} \mathrm{Y}^{-} \mathrm{YCl}_{3}$ in $0.1 \mathrm{M} \mathrm{HCl}$ ) was supplied by the Center for Advanced Biomedical Imaging at M..D Anderson Cancer Center. Approximately $45 \mathrm{MBq}$ of ${ }^{177} \mathrm{Lu}$ were incubated with $1.00 \mu \mathrm{g}$ of AABD in $50 \mu \mathrm{L}$ of ammonium acetate buffer $(0.1 \mathrm{M}$, $\mathrm{pH}$ 5.4) at $95^{\circ} \mathrm{C}$ for $15 \mathrm{~min}$, producing ${ }^{177} \mathrm{Lu}-\mathrm{AABD}$ in $99 \%$ decaycorrected radiochemical yield and $99 \%$ radiochemical purity as analyzed via radio-HPLC. ${ }^{86} \mathrm{Y}-\mathrm{AABD}$ was prepared via addition of approximately $50 \mu \mathrm{L}$ of as-received ${ }^{86} \mathrm{Y}^{-} \mathrm{YCl}_{3}(\sim 50 \mathrm{MBq})$ to $5 \mu \mathrm{g}$ of AABD in $50 \mu \mathrm{L}$ of ammonium acetate buffer $(0.1 \mathrm{M}, \mathrm{pH} 5.4)$ and subsequent incubation at $95^{\circ} \mathrm{C}$ for $15 \mathrm{~min}$. A radiochemical yield and radiochemical purity of more than $97.5 \%$ were achieved for ${ }^{86} \mathrm{Y}$ labeling. Neither ${ }^{86} \mathrm{Y}-\mathrm{AABD}$ nor ${ }^{177} \mathrm{Lu}-\mathrm{AABD}$ required additional purification after labeling. Because AABD itself does not bind DAbR1 (25), the relevant molar activities are those of ${ }^{177} \mathrm{Lu}-\mathrm{AABD}$ or ${ }^{86} \mathrm{Y}-\mathrm{AABD}$ and are equivalent to the molar activities of the as-received radionuclides (Supplemental Fig. 1; supplemental materials are available at http:// jnm.snmjournals.org).

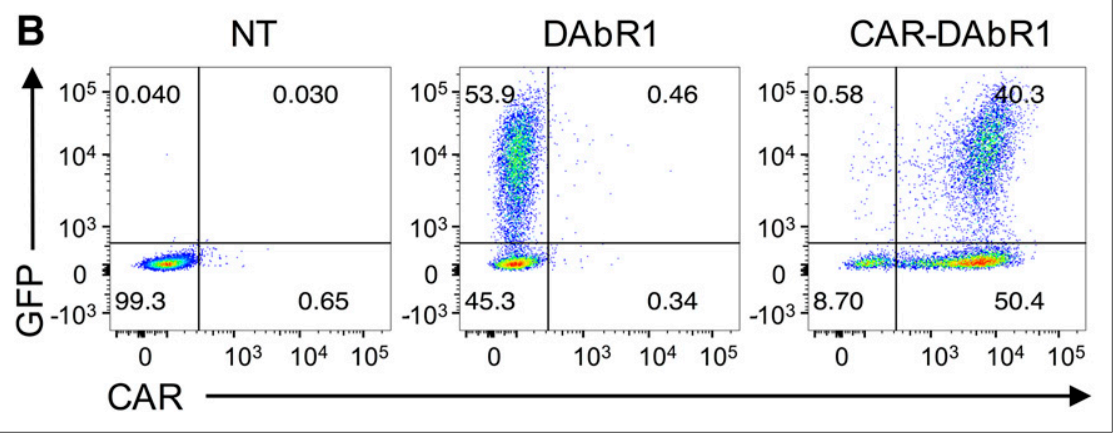

FIGURE 1. (A) Schematic structures of retroviral vectors SFG-DAbR1 and SFG-1928z (CAR). (B) Expression of DAbR1 (GFP) or CAR was confirmed using flow cytometry. Representative plots are shown.

\section{Determination of In Vitro Uptake of the Reporter Probes ${ }^{177} \mathrm{Lu} /{ }^{86}$ Y-AABD, Assessment of Cell Viability}

DAbR1 and nontransduced T cells $(250,000)$ were suspended in RPMI plus $10 \%$ fetal calf serum and incubated at $37^{\circ} \mathrm{C}$ with $3.7 \mathrm{kBq}$ of ${ }^{177} \mathrm{Lu} /{ }^{86} \mathrm{Y}-\mathrm{AABD}$ in $300 \mu \mathrm{L}$. Cells were harvested and counted in a WIZ$\mathrm{ARD}^{2}$ automatic $\gamma$-counter (PerkinElmer), and labeling efficiency (i.e., radioactivity 


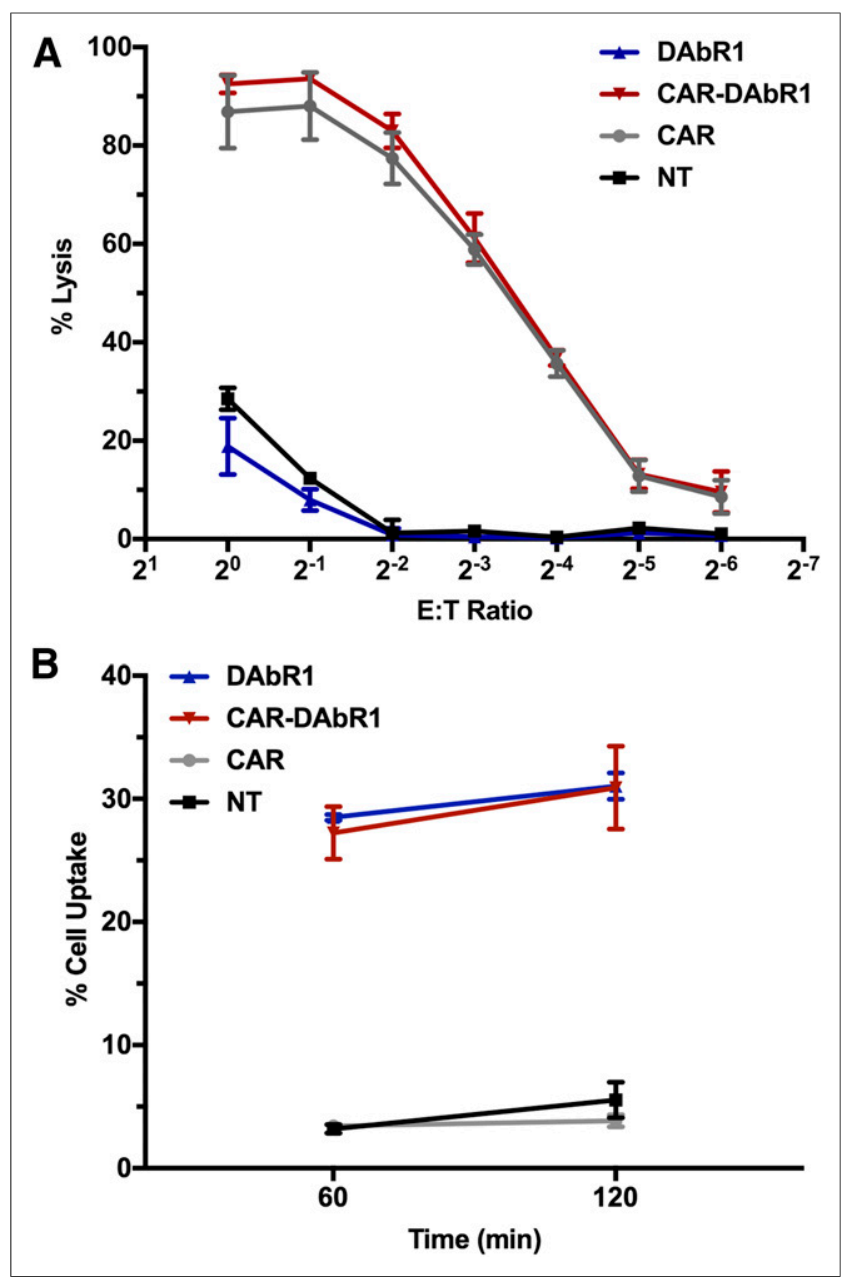

FIGURE 2. (A) In vitro standard cytotoxicity assay showing no difference in killing. One representative result is shown $(n=3)$. $\mathrm{E}=$ effector; $\mathrm{T}=$ target. (B) In vitro binding of ${ }^{86} \mathrm{Y}-\mathrm{AABD}$ at 1 and $2 \mathrm{~h}$. This representative dataset demonstrates specific binding of AABD to DAbR1-expressing T cells (DAbR1 and CAR-DAbR1), whereas no significant uptake was observed in nontransduced and CAR T cells. All experiments were performed in triplicate at $37^{\circ} \mathrm{C}$.

covalently bound to the cells) was calculated. Before and after cell uptake studies, cell viability was assessed by trypan blue exclusion testing.

\section{Animal Experiments: Xenograft Model to Assess Optimal Imaging Time-Point and Radiation Dose}

All animal experiments were performed under a protocol approved by MSK's Institutional Animal Care and Use Committee. U373.eGFP. firefly luciferase cells $\left(5 \times 10^{6}\right.$ in $\left.150 \mu \mathrm{L}\right)$ were injected subcutaneously over the right shoulder into ICR-SCID mice (IcrTac:ICR-Prkdc ${ }^{\text {scid; }}$; Taconic). Seven days later, the mice received subcutaneous implants of $\mathrm{T}$ cells over both shoulders (intratumorally on the right and subcutaneously on the left). For PET imaging studies, $3.5 \times 10^{6}$ DAbR1, CAR-DAbR1, and nontransduced T cells were suspended in $40 \mu \mathrm{L}$ of RPMI and subcutaneously injected; for SPECT imaging studies, $5 \times 10^{6}$ DAbR1 or $5 \times 10^{6}$ nontransduced T cells were implanted, followed $30 \mathrm{~min}$ later by intravenous radiotracer administration.

\section{Small-Animal PET/CT Imaging}

Small-animal PET/CT scans were performed using the Inveon PET/CT system (Siemens). Mice were anesthetized using 1.5\%-2\% isoflurane (Baxter Healthcare) and intravenously injected with ${ }^{86}$ Y-AABD (3.7 MBq). Ten-minute dynamic scans were initiated at the time of injection. At 2, 4, 16, and $24 \mathrm{~h}$, the same mice underwent 30-min static scans. Data were corrected for decay and detector dead time, and images were reconstructed by 2-dimensional ordered-subset expectation maximization into a $128 \times 128$ matrix $(0.78 \times 0.78 \times 0.80 \mathrm{~mm}$ voxel dimensions). Image counts per voxel per second were converted to activity concentrations $(\mathrm{Bq} / \mathrm{mL})$ using a system-specific calibration factor. CT scans were reconstructed using a modified Feldkamp cone-beam reconstruction algorithm to generate $512 \times 512 \times 768$ voxel image volumes $(0.197 \times 0.197 \times 0.197 \mathrm{~mm}$ voxel dimensions $)$.

\section{Image-Based Biodistribution and Organ Dosimetry of ${ }^{86}$ Y-AABD}

Quantification of organ-associated activity was performed via a combination of manual and semiautomatic segmentation techniques in 3D Slicer, version 4.6 (www.slicer.org), to define organ and target volumes. Organ activities were corrected for partial-volume effects using volume-dependent activity recovery coefficients derived from system-specific measurements of spheric phantoms containing aqueous ${ }^{86} \mathrm{Y}^{-} \mathrm{YCl}_{3}$.

Organ residence times were obtained via trapezoidal integration of the resulting time-activity curves, and the remaining residence time (i.e., after the last time point) was estimated using a monophasic exponential fit. The

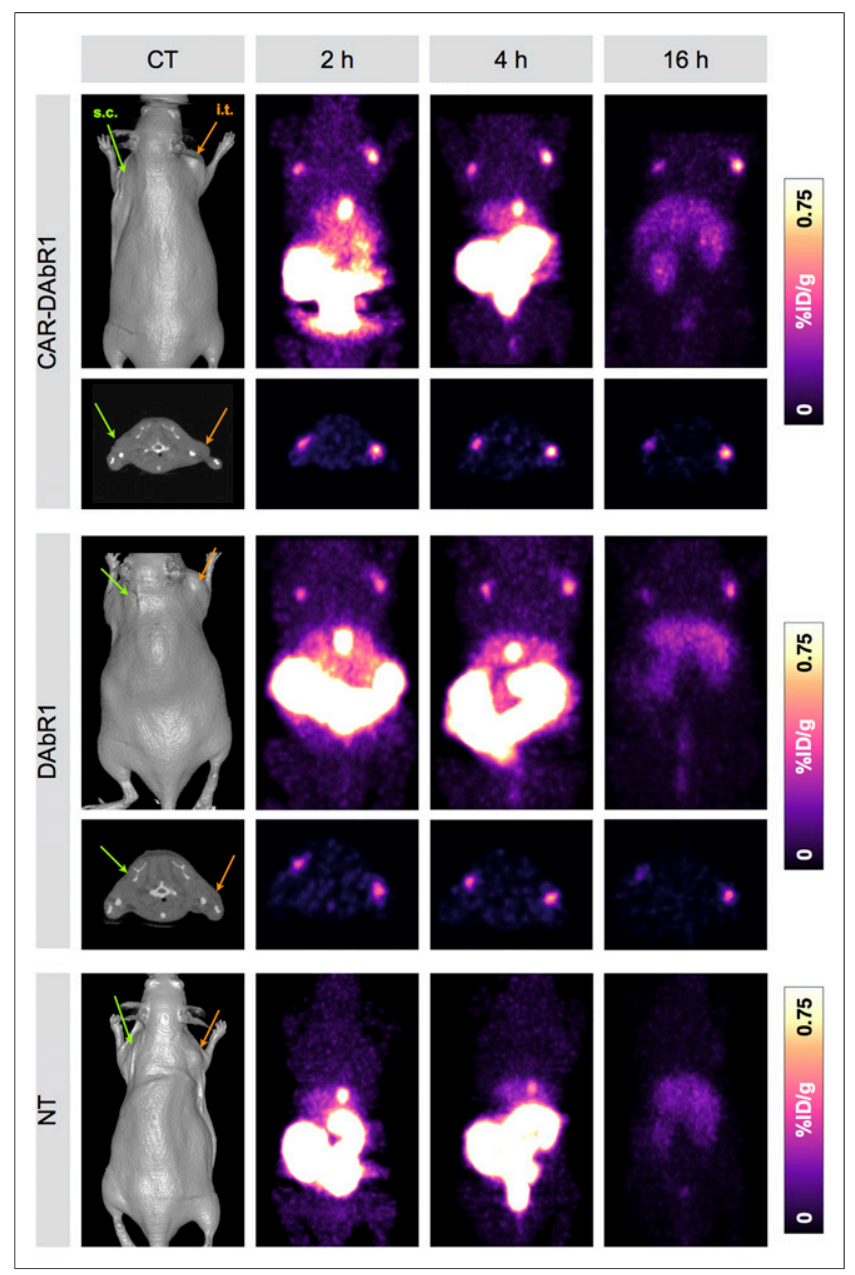

FIGURE 3. In vivo labeling after subcutaneous CAR-DAbR1, DAbR1, and NT T-cell implantation and serial imaging with PET/CT. Maximumintensity-projection images demonstrate uptake of ${ }^{86} \mathrm{Y}-\mathrm{AABD}$ into DAbR1-expressing T cell implants (right: intratumoral (i.t.), orange arrow; left: subcutaneous (s.c.), green arrow). Physiologic excreted tracer activity in kidneys, gallbladder, bowel, and urinary bladder on early imaging time points is markedly decreased on delayed imaging after $16 \mathrm{~h}$. 
CT-derived organ volumes were used to construct digital finite-element mesh phantoms via constrained Delaunay tetrahedralization using the mesh generation software Tetgen (26). These phantoms were subsequently implemented in the Monte Carlo transport code PHITS for absorbed dose calculations (supplemental materials). For human organ-level dose estimation, the human organ cumulated activities were derived and imported into OLINDA-EXM 1.0 to calculate mean organ absorbed doses and total-body effective dose in the 70-kg standard man model (Supplemental Table 1).

\section{SPECT/CT Imaging}

SPECT/CT scans were performed using a small-animal NanoSPECT/CT device from Mediso Medical Imaging Systems. Animals were injected with $37 \mathrm{MBq}$ of ${ }^{177} \mathrm{Lu}-\mathrm{AABD}$ in $200 \mu \mathrm{L}$ of phosphatebuffered saline via the tail vein. At 4 and $20 \mathrm{~h}$ after injection, the mice were anesthetized as described for PET/CT imaging, and SPECT images were acquired over $60 \mathrm{~min}$. Quantification was performed using a predetermined instrument calibration factor, and data were visualized in the InVivoScope integrated analysis software. SPECT data were corrected for decay and detector dead time and reconstructed by ordered-subset expectation maximization in a $256 \times 256$ matrix with a voxel size of $0.4 \mathrm{~mm}^{3}$ using the HiSPECT software package (Scivis $\mathrm{GmbH})$. CT scans were reconstructed with an isotropic voxel size of $0.147 \times 0.147 \times 0.147 \mathrm{~mm}$ using a Feldkamp cone-beam filteredbackprojection algorithm (Mediso Ltd.).

\section{Cell-Level Radiation Dosimetry of ${ }^{177}$ Lu-AABD}

T-cell-associated activity was quantified by drawing regions of interest around the entire tumor volume at $20 \mathrm{~h}$ after injection. SPECT images were corrected for background accumulation. A homogeneous activity distribution on the $\mathrm{T}$ cells was assumed, as well as negligible T-cell migration from the tumor and similar biologic clearance kinetics for ${ }^{86} \mathrm{Y}-\mathrm{AABD}$ and ${ }^{177} \mathrm{Lu}-\mathrm{AABD}$. The $\mathrm{T}$ cell and its nucleus were modeled as concentric spheres, with diameters of 12 and $8 \mu \mathrm{m}$, respectively. The absorbed dose for solitary $\mathrm{T}$ cells was estimated from cell surfaceto-nucleus ( $\mathrm{N} \leftarrow \mathrm{CS}$ ) self-dose $\mathrm{S}$ values obtained from MIRDcell (27).

\section{In Vivo Tracking of Adoptively Transferred T Cells}

NALM-6 cells expressing firefly luciferase-GFP $\left(10 \times 10^{6}\right.$ in $150 \mu \mathrm{L}$ ) were injected subcutaneously over the right shoulder into
NOD/SCID/IL-2R $\gamma$-null male mice (Jackson Laboratory). Seventeen days later, the mice were injected intravenously with $100 \mu \mathrm{L}$ of RPMI containing $3 \times 10^{6}$ DAbR1 T cells, CAR-DAbR1 T cells, or no T cells. On day 10 after T-cell injection, animals were injected with ${ }^{86}$ Y-AABD (3.7 MBq) or ${ }^{177} \mathrm{Lu}-\mathrm{AABD}(37 \mathrm{MBq})$ and imaged at 4 and $16 \mathrm{~h}$ (PET) or at 4 and $20 \mathrm{~h}$ (SPECT) after injection.

\section{Autoradiography and Immunohistochemical Staining}

To evaluate the localization of AABD in the tumor tissue and assess T-cell migration, NALM-6 tumors were harvested immediately after the last imaging scans and snap frozen in optimal-cutting-temperature compound (Tissue Tek). Tissue was cut into $10-\mu \mathrm{m}$ slices using cryostat sectioning. Coronal cryosections were exposed to a storage phosphor autoradiography plate (Fujifilm, BAS-MS2325) overnight at $-20^{\circ} \mathrm{C}$ for radiotracer localization and analyzed using ImageJ, version $1.47 \mathrm{u}$ (http://rsbweb.nih.gov/ij/).

Immunohistochemistry was performed on an automated immunostainer (Ventana Medical Systems). Staining was performed with primary antibodies against CD3 (DAKO) using the same cryosections. Appropriate positive and negative controls were used to confirm the adequacy of the staining. Adjacent sections were hematoxylin- and eosin-stained for morphologic evaluation.

\section{Statistics and Data Reporting}

All experimental data are presented as mean \pm SD. All in vitro experiments were performed in triplicate; Graph Pad Prism 7 software was used for statistical analysis. The differences between means were tested by appropriate tests, including the $t$ test. The significance level was a $P$ value of less than 0.05 .

\section{RESULTS}

\section{Characterization of DAbR1-Expressing T and CAR T Cells}

Transduction of DAbR1 and 1928z was confirmed by flow cytometry (Fig. 1B and Supplemental Fig. 2). Cell surface expression of the reporter gene was confirmed by flow cytometry using an anti $\mathrm{F}(\mathrm{ab})_{2}$ antibody (Supplemental Fig. 2). Transduction did not occur preferentially in any of the T-cell subtypes studied (Supplemental Fig. 3). Comparison of 1928z-DAbR1 (CAR-DAbR1) and 1928z (CAR) T cells did not demonstrate a difference in cytotoxic

TABLE 1

Mean Uptake in T-Cell Implants and T-Cell-to-Normal-Tissue Ratios (2 Mice) Derived from Serial ${ }^{86}$ Y-AABD PET/CT Imaging

\begin{tabular}{|c|c|c|c|c|c|c|c|c|}
\hline \multirow[b]{2}{*}{ Time (h) } & \multirow{2}{*}{$\begin{array}{c}\text { Tumor } \\
(\% \mathrm{ID} / \mathrm{g})\end{array}$} & \multicolumn{4}{|c|}{ Tumor } & \multicolumn{3}{|c|}{ Subcutaneous } \\
\hline & & TNR-blood & TNR-muscle & TNR-liver & Subcutaneous (\%ID/g) & TNR-blood & TNR-muscle & TNR-liver \\
\hline \multicolumn{9}{|l|}{ DAbR1 } \\
\hline 2 & 2.59 & 13.4 & 25.6 & 7.19 & 3.06 & 15.8 & 30.1 & 8.48 \\
\hline 4 & 4.04 & 22.0 & 59.9 & 11.4 & 4.00 & 21.8 & 59.3 & 11.2 \\
\hline 16 & 1.46 & 17.4 & 64.7 & 9.11 & 0.751 & 8.95 & 33.2 & 4.68 \\
\hline 24 & 1.36 & 19.3 & 28.5 & 6.96 & 0.736 & 10.47 & 15.5 & 3.78 \\
\hline \multicolumn{9}{|c|}{ CAR-DAbR1 } \\
\hline 2 & 2.28 & 11.3 & 19.1 & 6.68 & 1.54 & 7.61 & 12.9 & 4.51 \\
\hline 4 & 2.37 & 16.7 & 26.4 & 7.54 & 0.845 & 5.95 & 9.43 & 2.69 \\
\hline 16 & 1.82 & 28.0 & 85.1 & 12.1 & 1.09 & 16.6 & 50.6 & 7.20 \\
\hline 24 & 1.54 & 20.1 & 42.5 & 9.33 & 1.33 & 17.3 & 36.6 & 8.02 \\
\hline
\end{tabular}




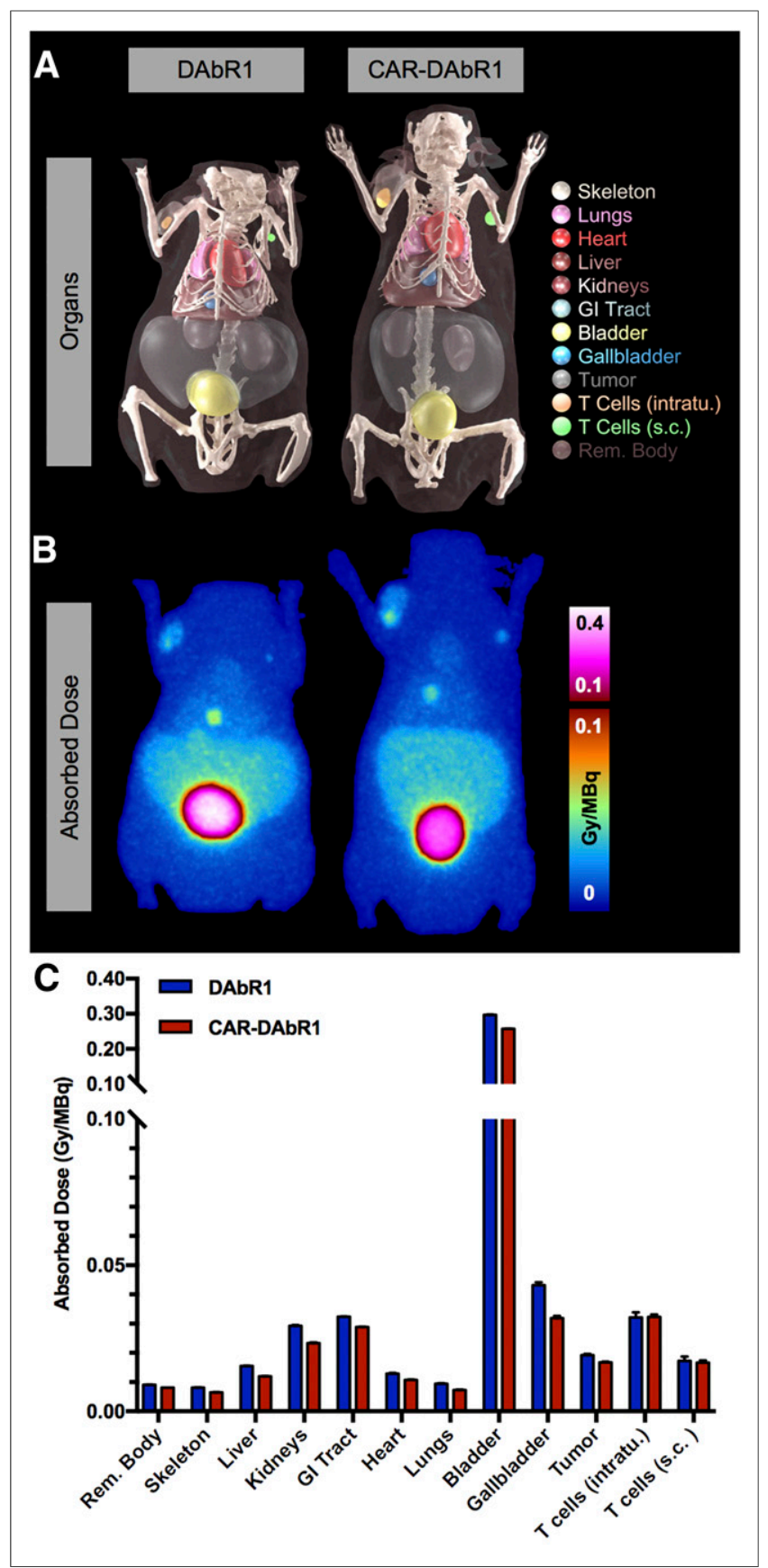

FIGURE 4. Radiation dosimetry. (A) CT-derived FEM phantom used in PHITS absorbed dose calculations. (B) Absorbed dose map (maximum-intensity projection; $0.45-\mathrm{mm}$ isotropic voxel size) simulated in PHITS for ${ }^{86} Y$-AABD PET imaging of DAbR1 and CAR-DAbR1 T cells in U373 mouse model. (C) Organ-level average dose to T cells and normal tissues. $\mathrm{Gl}=$ gastrointestinal; intratu. $=$ intratumoral; s.c. $=$ subcutaneous; $\mathrm{T}=$ tumor.

effector functions (Fig. 2A). For further in vitro studies, DAbR1 and CAR DAbR1 T cells were purified for DAbR1 expression using the magnetic activated cell-sorting-based cell sorting after staining for anti-F(ab) $)_{2}$ (Supplemental Fig. 4).

In in vitro binding assays, both DAbR1 and CAR-DAbR1 $\mathrm{T}$ cells exhibited high accumulation of ${ }^{86} \mathrm{Y}-\mathrm{AABD}$, whereas the percentage uptake of the nontransduced $\mathrm{T}$ cells remained low (Fig. 2B).
Comparable ratios were observed after incubation with ${ }^{177} \mathrm{Lu}-\mathrm{AABD}$ (data not shown). Thus, transduced $\mathrm{T}$ cells expressed membrane-bound $\mathrm{DAbR} 1$, enabling specific binding of radiolabeled AABD in vitro.

\section{In Vivo Imaging and Radiation Dosimetry}

PET/CT scans already demonstrated focal uptake at the site of T-cell injection $2 \mathrm{~h}$ after injection $(2.37 \pm 0.63$ percentage injected dose [\%ID]/g) (Fig. 3; Table 1; Supplemental Table 2). Uptake persisted for the intratumorally injected cells, whereas activity decreased at the subcutaneous T-cell injection site. The biodistribution in normal organs was as described previously, with rapid clearance of the activity to kidneys and bowel. The highest absolute uptake was observed in T-cell implants at $4 \mathrm{~h}$, whereas the highest T-cell-to-normalbackground contrast was observed at $16 \mathrm{~h}$ after injection (Table 1).

Organ residence times calculated from tissue uptake data (Supplemental Figs. 5A and 5B) were used to estimate radiation dose in mice (Fig. 4) and humans (Supplemental Table 1). The highest radiation doses were observed for the urinary bladder wall, followed by the gallbladder, small and large intestines, and kidneys.

SPECT imaging studies with ${ }^{177} \mathrm{Lu}-\mathrm{AABD}$ also demonstrated focal uptake at the site of DAbR1 T cell injection (Supplemental Fig. 6).

Intratumoral T-cell-associated activity at $20 \mathrm{~h}$ after injection of ${ }^{177} \mathrm{Lu}-$ AABD (Supplemental Fig. 7) was $0.0013 \mathrm{MBq} / \mathrm{MBq}$ injected, yielding a maximum T-cell-absorbed dose of $1.1 \mathrm{~Gy} / \mathrm{MBq}$ for highly clustered cells. The solitary T-cell dose was computed as 0.015 Gy/MBq.

\section{In Vivo Tracking of Adoptively Transferred T Cells}

PET/CT scans demonstrated heterogeneously increased uptake indicating homing of CAR-DAbR1 T cells at the tumor site (Fig. 5). No uptake above background at the tumor site was seen in mice receiving DAbR1 $\mathrm{T}$ cells or no $\mathrm{T}$ cells at all (Fig. 5 and Supplemental Fig. 8). Migration of targeted $\mathrm{T}$ cells to the tumor was also confirmed on SPECT/CT (Fig. 6A).

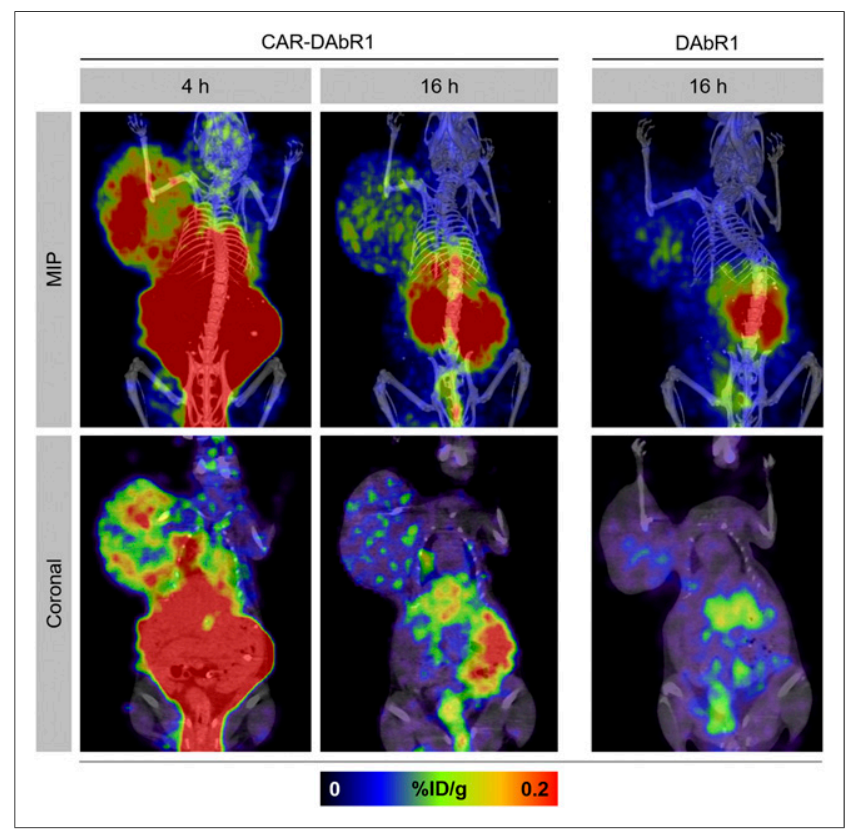

FIGURE 5. In vivo tracking of adoptively transferred T cells with PET. Maximum-intensity-projection (MIP) images and selected coronal PET/CT images at 4 and $16 \mathrm{~h}$ after injection depicting homing and accumulation of CAR-DAbR1 $\mathrm{T}$ cells at tumor site over right shoulder. No uptake above background at tumor site is noted after DAbR1 T-cell administration. 


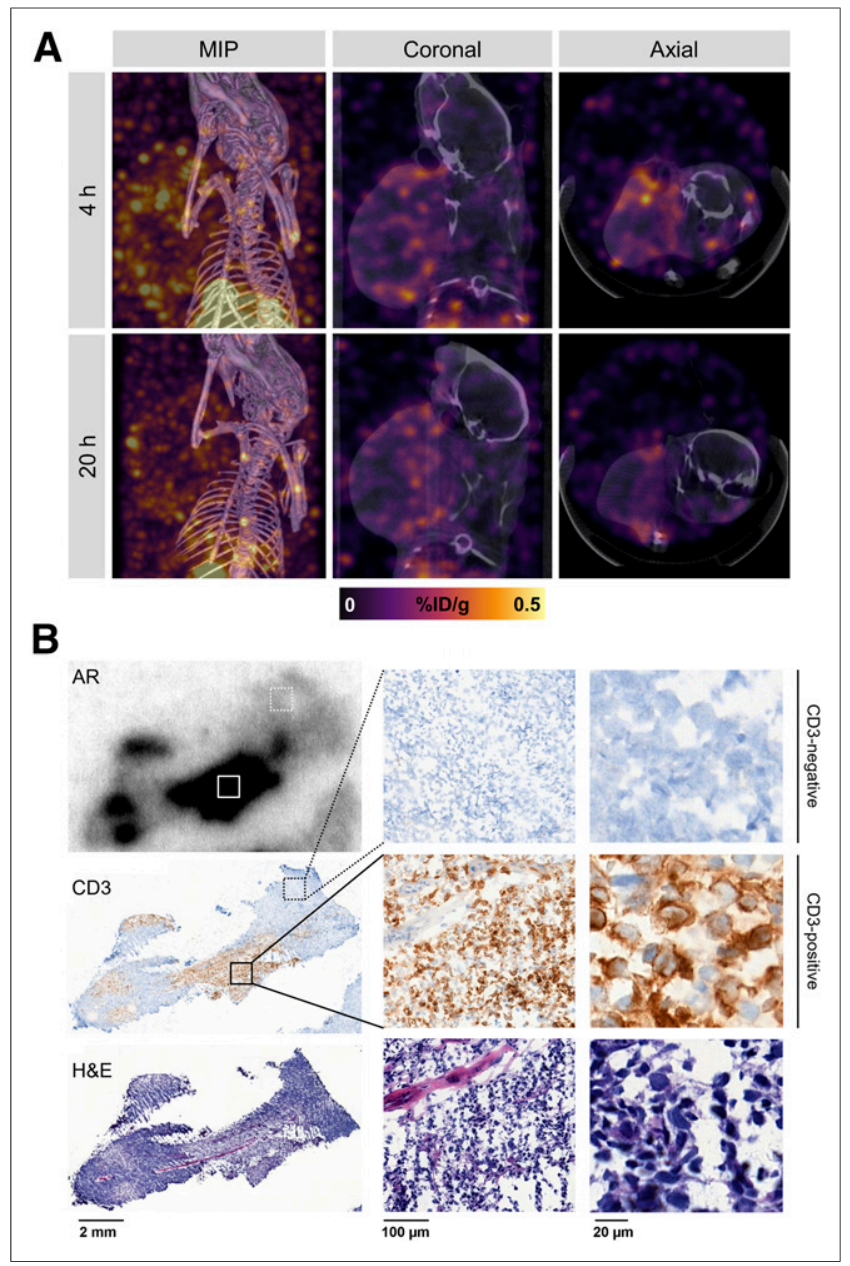

FIGURE 6. Homing of CAR-DAbR1 T cells to tumor is visualized on SPECT/CT and confirmed on autoradiography and immunohistochemistry. (A) Maximum-intensity-projection (MIP), coronal, and axial images at 4 and $20 \mathrm{~h}$ after injection demonstrating uptake of ${ }^{177} \mathrm{Lu}-\mathrm{AABD}$ into CAR-DAbR1 T cells localized in tumor. (B) Autoradiography (AR) and immunohistochemistry of tumor tissue containing CAR-DAbR1 T cells. Radiotracer colocalizes with CD3-positive T-cell cluster (brown staining). $\mathrm{H} \& \mathrm{E}=$ hematoxylin and eosin.

\section{Autoradiography and Immunohistochemistry of CAR-DAbR1 T Cell Homing}

Autoradiography and CD3 staining of tumor tissue indicated that the areas with the highest activity concentration were those infiltrated by CAR-DAbR1 T cells (Fig. 6B).

\section{DISCUSSION}

We present a novel imaging tool to track genetically engineered $\mathrm{T}$ cells in vivo using PET. We demonstrated that DOTA-antibody reporter DAbR1 can be expressed on lymphocytes and CAR T cells. Also, DAbR 1 coexpression does not interfere with cytotoxic effector functions of tumor-specific $1928 \mathrm{z}$ (CAR) $\mathrm{T}$ cells in vitro.

The advantages of the described reporter gene/reporter probe combination are that the reporter probe is based on a molecule (DOTA) that has been used clinically as part of MRI contrast agents and radiopharmaceuticals. Also, the intrinsic properties of the DAbR1/AABD reporter gene/reporter probe system, characterized by the infinite affinity component, potentially facilitates detection of the DAbR1-expressing cells as it allows persistent (covalent) binding of the probe at the target site while unbound tracer is cleared, thus improving contrast. Indeed, the probes, ${ }^{177} \mathrm{Lu}-\mathrm{AABD}$, and ${ }^{86} \mathrm{Y}-\mathrm{AABD}$, remained bound to DAbR1-expressing lymphocytes beyond $24 \mathrm{~h}$, whereas the unbound probe continuously cleared from normal organs, resulting in excellent T-cell-to-background ratios and high-contrast images.

Our observed in vitro uptake of radiolabeled AABD to DAbR1expressing T cells $\left({ }^{86} \mathrm{Y}-\mathrm{AABD}\right.$ at $2 \mathrm{~h}: 31.04 \% \pm 1.8 \%$ for DAbR1 and $30.91 \% \pm 5.8 \%$ for CAR-DAbR 1 per $2.5 \times 10^{5}$ cells) compares favorably with prior data using other reporter genes, such as hNET, human sodium iodide symporter, HSV-tk, and human deoxycytidine kinase double mutant (12). Moroz et al. (12) reported that the highest in vitro uptake levels were with ${ }^{123}$ I-metaiodobenzylguanidine, ${ }^{124}$ I-metaiodobenzylguanidine, and ${ }^{18} \mathrm{~F}$-metafluorobenzylguanidine in hNET reporter-bearing $\mathrm{T}$ cells after $2 \mathrm{~h}$ of incubation $(6.5 \% \pm$ $0.4 \%, 7.6 \% \pm 0.1 \%$, and $1.9 \% \pm 0.2 \%$ of added radioactivity per $10^{6}$ cells, respectively), followed by HSV-tk $\left(2^{\prime}{ }^{18}{ }^{18}\right.$-fluoro-5-ethyl1-beta-D-arabinofuranosyluracil $\left[{ }^{18} \mathrm{~F}-\mathrm{FEAU}\right]: 4.1 \% \pm 0.41 \%$ per $10^{6}$ cells), human sodium iodide symporter (with ${ }^{124}$ I-iodide: $2.2 \% \pm$ $0.9 \%$ per $10^{6}$ cells), and human deoxycytidine kinase double mutant (124I-fialuridine: $1.5 \% \pm 0.25 \%$ per $10^{6}$ cells) reporter-bearing $\mathrm{T}$ cells. In subsequent in vivo PET imaging studies of subcutaneously implanted reporter-bearing T cells $\left(3 \times 10^{6}\right)$, T-cell-to-

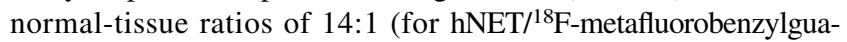
nidine at $4 \mathrm{~h}$ ) and 7:1 (for hNET/ ${ }^{124}$ I-metaiodobenzylguanidine at $24 \mathrm{~h}$ ) were noted, both of which are lower than the observed ratios for the DAbR1/ ${ }^{86}$ Y-AABD reporter system (38.7:1 at $4 \mathrm{~h}$ and 30.8:1 at $24 \mathrm{~h}$; Supplemental Table 2).

Najjar et al. (28) incorporated HSV-tk, firefly luciferase, and CD19-specific CAR using the non-virus-based "sleeping beauty" transposon/transposase system into primary human $\mathrm{T}$ cells and assessed in vitro and in vivo labeling with ${ }^{18}$ F-FEAU. Similar to our results, investigators reported unaltered cytotoxic effector function of the reporter gene bearing CAR T cells in in vitro cytotoxicity assays. In vitro uptake of ${ }^{18} \mathrm{~F}-\mathrm{FEAU}$ led to a cell-to-medium accumulation ratio of 16.8 at $2 \mathrm{~h}$. Control T cells were not included, limiting direct comparison with the current study. For in vivo labeling, $7.5 \times 10^{6}$ and $15 \times 10^{6}$ CAR-positive, TK-positive, firefly luciferase-positive $\mathrm{T}$ cells were subcutaneously injected, which is about a 2- and 4-fold higher T-cell number than in the presented study. PET imaging at $2 \mathrm{~h}$ after injection of $3.7 \mathrm{MBq}$ of ${ }^{18} \mathrm{~F}-\mathrm{FEAU}$ confirmed uptake in T-cell implants yielding about $5 \% \mathrm{ID} / \mathrm{g}$, which is similar to what we observed in our study. However, a disadvantage of the HSV-tk reporter gene is the immunogenicity, which is likely to affect $\mathrm{T}$-cell function in vivo.

The dosimetry of ${ }^{86} \mathrm{Y}-\mathrm{AABD}$ determined in our study is comparable to that of ${ }^{124}$ I-fialuridine (29), which has been used in clinical studies to image HSV-tk reporter gene expression. Previous data suggest that $\mathrm{T}$ lymphocytes can tolerate absorbed radiation doses of up to about 830 cGy (median value) (30). Higher doses may result in radiation damage, impaired immune function, and reduced cell proliferation. According to our data, the mean absorbed dose to the $\mathrm{T}$ cells by systemic administration of $3.7 \mathrm{MBq}$ of ${ }^{86} \mathrm{Y}-\mathrm{AABD}$ is in the range of $20 \mathrm{cGy}$-well below the threshold for immune cytotoxicity. Thus, repeated imaging studies for monitoring of T cells' fate over time appear feasible.

Because adoptive cell therapy is potentially associated with lifethreatening side effects, we explored the use of DAbR1 as a suicide gene. Indeed, use of the therapeutic lanthanide ${ }^{177} \mathrm{Lu}$ resulted in an absorbed dose of $1.1 \mathrm{~Gy} / \mathrm{MBq}$ for highly clustered $\mathrm{T}$ cells in mice, 
markedly above the aforementioned threshold and thus likely to result in at least impaired if not ablated T-cell function.

However, our calculations of the radiation dose to $\mathrm{T}$ cells are first-order approximations because we considered only clusters of tightly packed $\mathrm{T}$ cells and assumed no migration of $\mathrm{T}$ cells during the decay of the isotope. Future experimental studies are needed to clarify potential therapeutic applications of ${ }^{177} \mathrm{Lu}-\mathrm{AABD}$.

To demonstrate the utility of the DAbR1/AABD reporter system to monitor adoptively transferred $\mathrm{T}$ cells, we investigated the migration of CD19-targeted CAR T cells after tail vein injection in a subcutaneous CD19-positive tumor mouse model. Using PET (and SPECT) imaging, we confirmed successful homing of CAR $\mathrm{T}$ cells to the tumor site. The imaging findings were corroborated in ex vivo autoradiographic and immunohistochemical analyses.

We are aware that our study has limitations. First, the murinederived single-chain Fv fragment used in the DAbR1 may induce immune reactions in humans. However, humanized or fully human single-chain Fv fragments could reduce the risk of immunogenicity for clinical applications. Furthermore, structural revision could also address the need and optimal length for a hinge.

\section{CONCLUSION}

We have presented a novel T-cell-tracking approach based on a DOTA-lanthanoid antibody reporter with infinite affinity. The high uptake and the prolonged and specific binding of radiolabeled AABD to the transduced T cells, and the low potential for immunogenicity, render DAbR1/AABD a promising tool for monitoring the dynamics of adoptive cell therapies.

\section{DISCLOSURE}

This research was supported by NIH/NCI Cancer Center Support Grant P30 CA008748, NIH grant S10 RR028889-01, and NIH grant S10 OD016207-01 (MSK Small-Animal Imaging Core), as well as the NIH/NCI Paul Calabresi Career Development Award for Clinical Oncology K12 CA184746 (to Simone Krebs) and the Center for Targeted Radioimmunotherapy and Theranostics of the Ludwig Center for Cancer Immunotherapy. Lukas M. Carter acknowledges support from the Ruth L. Kirschstein postdoctoral fellowship (NIH F32-EB025050). No other potential conflict of interest relevant to this article was reported.

\section{ACKNOWLEDGMENTS}

We gratefully acknowledge the staff members at the MSK SmallAnimal Imaging Core (Valerie Longo and Pat Zanzonico), Jason Lewis, and Hanwen Zhang for helpful discussions and support, and Leah Bassity for editing assistance. We wish especially to thank Liu $\mathrm{H}$. Wei at UCLA for providing materials and helpful guidance.

\section{REFERENCES}

1. Rosenberg SA, Restifo NP, Yang JC, Morgan RA, Dudley ME. Adoptive cell transfer: a clinical path to effective cancer immunotherapy. Nat Rev Cancer. 2008;8:299-308.

2. Sadelain M, Riviere I, Riddell S. Therapeutic T cell engineering. Nature. 2017;545:423-431

3. Krebs S, Rodriguez-Cruz TG, Derenzo C, Gottschalk S. Genetically modified T cells to target glioblastoma. Front Oncol. 2013;3:322.

4. Sadelain M. CAR therapy: the CD19 paradigm. J Clin Invest. 2015;125:3392-3400.

5. Porter DL, Levine BL, Kalos M, Bagg A, June CH. Chimeric antigen receptormodified T cells in chronic lymphoid leukemia. N Engl J Med. 2011;365:725-733.
6. Brentjens R, Yeh R, Bernal Y, Riviere I, Sadelain M. Treatment of chronic lymphocytic leukemia with genetically targeted autologous $\mathrm{T}$ cells: case report of an unforeseen adverse event in a phase I clinical trial. Mol Ther. 2010;18:666668.

7. Teachey DT, Lacey SF, Shaw PA, et al. Identification of predictive biomarkers for cytokine release syndrome after chimeric antigen receptor T-cell therapy for acute lymphoblastic leukemia. Cancer Discov. 2016;6:664-679.

8. Yaghoubi SS, Campbell DO, Radu CG, Czernin J. Positron emission tomography reporter genes and reporter probes: gene and cell therapy applications. Theranostics. 2012;2:374-391.

9. McCracken MN, Vatakis DN, Dixit D, McLaughlin J, Zack JA, Witte ON. Noninvasive detection of tumor-infiltrating $\mathrm{T}$ cells by PET reporter imaging. $J$ Clin Invest. 2015;125:1815-1826.

10. Keu KV, Witney TH, Yaghoubi S, et al. Reporter gene imaging of targeted T cell immunotherapy in recurrent glioma. Sci Transl Med. 2017;9:eaag2196.

11. Riddell SR, Elliott M, Lewinsohn DA, et al. T-cell mediated rejection of genemodified HIV-specific cytotoxic T lymphocytes in HIV-infected patients. Nat Med. 1996;2:216-223.

12. Moroz MA, Zhang H, Lee J, et al. Comparative analysis of $\mathrm{T}$ cell imaging with human nuclear reporter genes. J Nucl Med. 2015;56:1055-1060.

13. Doubrovin MM, Doubrovina ES, Zanzonico P, Sadelain M, Larson SM, O'Reilly RJ. In vivo imaging and quantitation of adoptively transferred human antigen-specific $\mathrm{T}$ cells transduced to express a human norepinephrine transporter gene. Cancer Res. 2007;67:11959-11969.

14. Zhang H, Moroz MA, Serganova I, et al. Imaging expression of the human somatostatin receptor subtype-2 reporter gene with ${ }^{68} \mathrm{Ga}$-DOTATOC. J Nucl Med. 2011;52:123-131.

15. Goodwin DA, Meares CF, Watanabe N, et al. Pharmacokinetics of pretargeted monoclonal antibody 2D12.5 and ${ }^{88}$ Y-Janus-2-(p-nitrobenzyl)-1,4,7,10-tetraazacyclododecanetetraacetic acid (DOTA) in BALB/c mice with KHJJ mouse adenocarcinoma: a model for ${ }^{90} \mathrm{Y}$ radioimmunotherapy. Cancer Res. 1994;54: 5937-5946.

16. Lubic SP, Goodwin DA, Meares CF, Song C, Osen M, Hays M. Biodistribution and dosimetry of pretargeted monoclonal antibody 2D12.5 and Y-Janus-DOTA in BALB/c mice with KHJJ mouse adenocarcinoma. J Nucl Med. 2001;42:670678.

17. Corneillie TM, Whetstone PA, Lee KC, Wong JP, Meares CF. Converting weak binders into infinite binders. Bioconjug Chem. 2004;15:1389-1391.

18. Corneillie TM, Lee KC, Whetstone PA, Wong JP, Meares CF. Irreversible engineering of the multielement-binding antibody 2D12.5 and its complementary ligands. Bioconjug Chem. 2004;15:1392-1402.

19. Aweda TA, Beck HE, Wu AM, Wei LH, Weber WA, Meares CF. Rates and equilibria for probe capture by an antibody with infinite affinity. Bioconjug Chem. 2010;21:784-791.

20. Wei LH, Olafsen T, Radu C, et al. Engineered antibody fragments with infinite affinity as reporter genes for PET imaging. J Nucl Med. 2008;49:1828-1835.

21. Brentjens RJ, Latouche JB, Santos E, et al. Eradication of systemic B-cell tumors by genetically targeted human T lymphocytes co-stimulated by CD80 and interleukin-15. Nat Med. 2003;9:279-286.

22. Gong MC, Latouche JB, Krause A, Heston WD, Bander NH, Sadelain M. Cancer patient $T$ cells genetically targeted to prostate-specific membrane antigen specifically lyse prostate cancer cells and release cytokines in response to prostatespecific membrane antigen. Neoplasia. 1999;1:123-127.

23. Krenciute G, Krebs S, Torres D, et al. Characterization and functional analysis of scFv-based chimeric antigen receptors to redirect $\mathrm{T}$ cells to IL13Ralpha2positive glioma. Mol Ther. 2016;24:354-363.

24. Eyquem J, Mansilla-Soto J, Giavridis T, et al. Targeting a CAR to the TRAC locus with CRISPR/Cas9 enhances tumour rejection. Nature. 2017;543:113-117.

25. Corneillie TM, Whetstone PA, Lee KC, Wong JP, Meares CF. Converting weak binders into infinite binders. Bioconjug Chem. 2004;15:1389-1391.

26. Wang Y, Helminen E, Jiang J. Building a virtual simulation platform for quasistatic breast ultrasound elastography using open source software: a preliminary investigation. Med Phys. 2015;42:5453-5466.

27. Vaziri B, Wu H, Dhawan AP, Du P, Howell RW. MIRD pamphlet no. 25: MIRDcell V2.0 software tool for dosimetric analysis of biologic response of multicellular populations. J Nucl Med. 2014;55:1557-1564.

28. Najjar AM, Manuri PR, Olivares S, et al. Imaging of sleeping beauty-modified CD19-specific T cells expressing HSV1-thymidine kinase by positron emission tomography. Mol Imaging Biol. 2016;18:838-848.

29. Jacobs A, Braunlich I, Graf R, et al. Quantitative kinetics of $\left[{ }^{124} \mathrm{I}\right] \mathrm{FIAU}$ in cat and man. J Nucl Med. 2001;42:467-475.

30. Zanzonico P, Koehne G, Gallardo HF, et al. [ $\left.{ }^{131} \mathrm{I}\right]$ FIAU labeling of genetically transduced, tumor-reactive lymphocytes: cell-level dosimetry and dose-dependent toxicity. Eur J Nucl Med Mol Imaging. 2006;33:988-997. 